\title{
Nodular Mucinosis of Breast: A Case Report
}

\author{
Tangul Bulut ${ }^{\mathrm{a}}$, Betul Celik ${ }^{\mathrm{a}, \mathrm{d}}$, Aziza Nassar ${ }^{\mathrm{b}}$, Arzu Didem Yalcin ${ }^{\mathrm{c}}$
}

\begin{abstract}
Nodular mucinosis (NM) is a dermal benign lesion which is presented as an acellular mucinous pool in the subcutaneous tissue. It is seen very rarely in the breast and when a mucinous pool is detected in the breast mass, primary mucinous neoplasms of the breast are the first in the differential and this lesion is often missed. Here we report an NM case in a breast and present its radiologic, clinical and pathological features and discuss its differential diagnosis.
\end{abstract}

Keywords: Nodular; Mucinosis; Breast; Mucin

\section{Introduction}

Nodular mucinosis (NM) of the breast is a very rare benign lesion. It is distinct from myxomas developed in Carney complex. There are 13 reported cases in the English literature [19]. All the reported cases involve around the nipple of young women, as well as men. The myxoid appearance of this lesion is due to abundant stromal acid mucopolysaccharide, which stains strongly positive with Alcian blue. It is a benign lesion with complete surgical excision.

Herein we reported a case of a woman who presented with pain and mass lesion in her right breast along with its radiological and pathological findings.

\section{Case Report}

The patient was a 37-year-old female without any relevant

Manuscript accepted for publication August 13, 2015

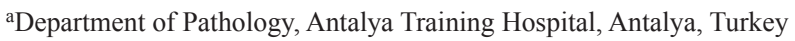
bLaboratory medicine and Pathology, Mayo Clinic, Jacksonville, FL, USA 'Internal Medicine, Allergy and Clinical Immunology, Antalya Training Hospital, Antalya, Turkey and Genomics Research Center, Academia Sinica, Taipei, Taiwan

${ }^{\mathrm{d}}$ Corresponding Author: Betul Celik, Department of Pathology, Antalya Training Hospital, Varlik Mahallesi Kazim Karabekisr Cad, Antalya 07100, Turkey. Email: bet_celik@yahoo.com

doi: http://dx.doi.org/10.14740/jmc2271w personal or family history who was admitted to the hospital for a mass lesion and pain in her right breast. Physical examination revealed a mass lesion at the 8 - 9 o'clock position, adjacent to the areola with a soft consistency. There were no palpable axillary lymph nodes. Ultrasonography demonstrated a $36 \times 12 \mathrm{~mm}$ hypoechoic heterogenic mass that is 3 $\mathrm{mm}$ away from the skin. It also had multiple lobulation on its posterior side. There were also cystic areas at the periphery of the lesion; however, there was no internal vascularity within the mass. Differential diagnosis included a solid parenchymal lesion, such as phylloides tumor or abscess. Patient was informed and an excisional biopsy of the mass lesion was done for diagnostic purpose.

Pathologic examination of the excised lesion revealed a $3.2 \times 2.5 \times 1.7 \mathrm{~cm}$ nodular myxoid mass. Histopathological examination of the mass revealed well-circumscribed multifocal mucinous pools within the breast parenchyma (Fig. 1). There was neither epidermal connection nor demonstrable epithelial cell component (Fig. 2). Mucinous pools were hypocellular, with rare CD68-positive histiocytes (Fig. 3), and congested vessels. There was neither estrogen nor progesterone receptors' immunoexpression. The mucinous pools were stained with Alcian blue ( $\mathrm{pH}$ 2.5) histochemical stain (Fig. 4). The patient was free of any recurrence after 29 months of clinical/ radiological follow-up.

\section{Discussion}

$\mathrm{NM}$ of the breast is a very rare benign stromal tumor. Although the term was initially described by Michal et al [2], the first case had been reported under the name of "nerve sheath myxoma of the breast" by Wee et al [1]. It is distinct from widespread mucinous nodules that can be seen in the breast as a component of Carney complex syndrome, and always seen around the areola or nipple complex.

Most cases are seen in young women, but three cases were reported in men and another case in a 72-year-old woman. The lesion grows slowly with a soft consistency. It is located superficially. Ultrasonography reveals a well-circumscribed lobular mass without microcalcification or any spicular extensions. Usually the lesion has lobular configuration and consists of mucinous pools within the breast parenchyma without benign or malignant epithelial cell component. Breast ducts can be 


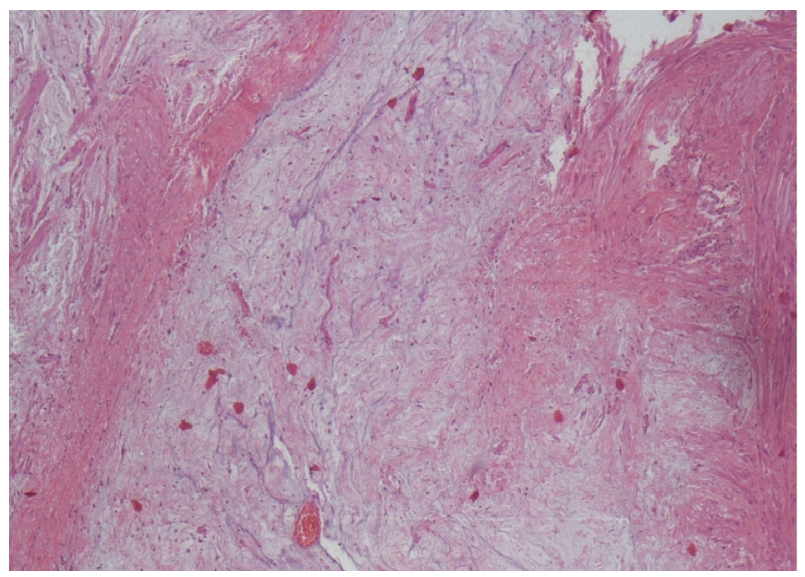

Figure 1. There is hypocellular well-circumscribed mucinous pool in the breast paranchyma $(\mathrm{H} \& \mathrm{E}, \times 4)$.

seen between the mucinous lobules but not within the pools [2]. Vessels and some benign-looking spindle stromal cells which may stain with smooth muscle actin (SMA), and CD68positive histiocytes may also be seen. The myxoid background is composed of acid mucopolysaccharide and stains with Alcian blue and Hale's colloidal iron. It is usually negative with periodic acid Schiff (PAS) stain.

Histogenesis of NM is unknown. Several investigators consider NM as a tumor of primitive mesenchymal cells [4]. Rosen proposed NM as a variant of myofibroblastoma, since few spindle cells within the lesion had the characteristic of myofibroblasts in his two reported cases [5]. Although Sanati et al did not claim the same histogenesis in their reported case [4], Rosen accepted SMA immunopositivity and smooth muscle myosin and S-100 immunonegativity of Sanati's case as a finding that supported his claim for a myofibroblastic origin [5].

Another proposal is that NM is a soft tissue tumor within the spectrum of myofibroblastoma and spindle cell lipoma, since both myofibroblastoma and spindle cell lipoma share

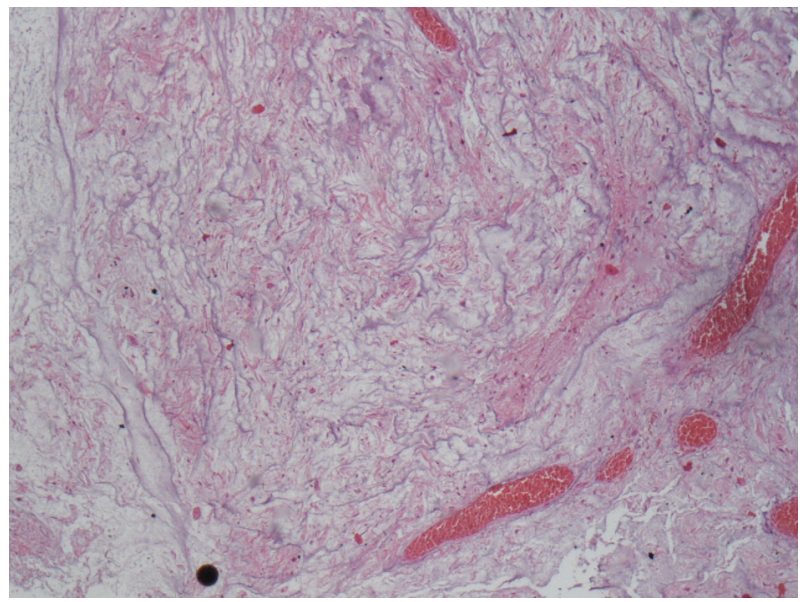

Figure 2. Mucinous lesion has no epithelial cell. Bottom right corner shows congested vessels $(\mathrm{H} \& \mathrm{E}, \times 10)$.

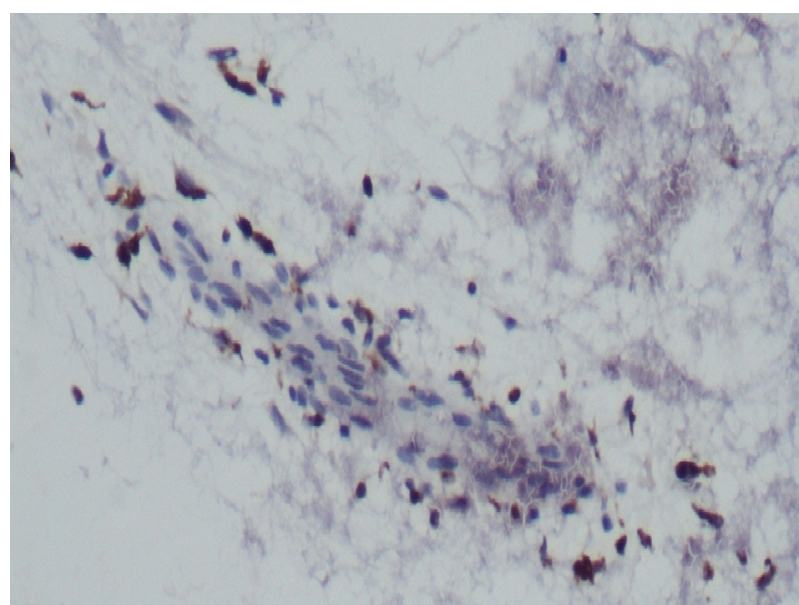

Figure 3. Histiocytes are highlighted by CD68 immunostain $(\times 40)$.

many histologic features, including genetic aberrations [9]. This hypothesis emanates from the immunohistochemical findings of diffuse CD34 and weak but noticeable S-100 immunopositivity of the case.

Although many breast lesions are included in the differential diagnosis, existence and presence of epithelial cells' component in the mucinous pool of those lesions excluded them easily without immunostains (e.g. myxoid fibroadenoma, mucinous DCIS, mucinous/colloid carcinoma and micropapillary carcinoma in situ). Moreover, mucocele-like lesions with associated carcinoma have coarse calcification radiologically than benign mucocele-like lesions and mucinous material in the former lesion is PAS-positive [10].

The main differential diagnosis of NM is trauma-related mucinosis [11].

In conclusion, this is a benign disease with no evidence of recurrence upon complete surgical excision of the lesion; however, since this is a very rare lesion, malignant mucinous breast lesion has not to be recognized incorrectly as NM, which may lead to inadequate treatment.

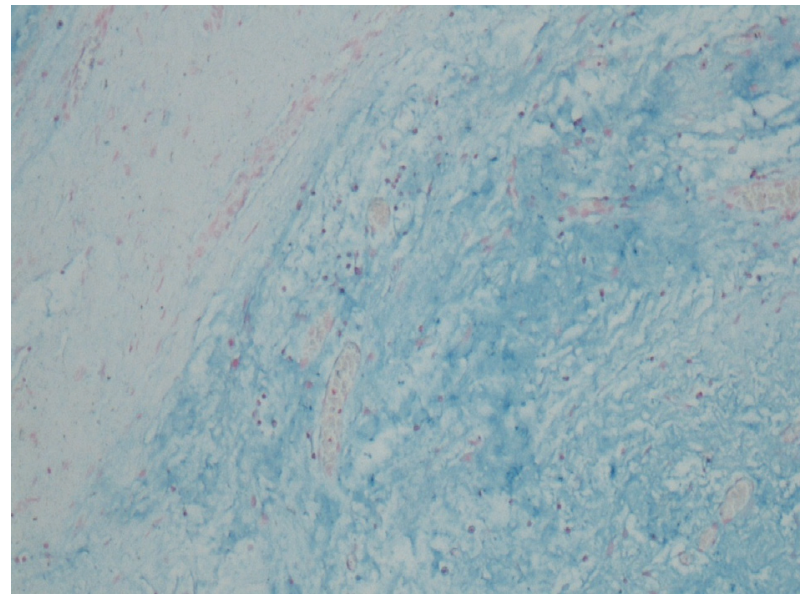

Figure 4. Alcian blue histochemical stain demonstrates acid mucopolysaccharide within mucinous pool (Alcian blue, $\times 20$ ). 


\section{Conflict of Interest}

None.

\section{References}

1. Wee A, Tan CE, Raju GC. Nerve sheath myxoma of the breast. A light and electron microscopic, histochemical and immunohistochemical study. Virchows Arch A Pathol Anat Histopathol. 1989;416(2):163-167.

2. Michal M, Ludvikova M, Zamecnik M. Nodular mucinosis of the breast: report of three cases. Pathol Int. 1998;48(7):542-544.

3. Koide N, Akashi-Tanaka S, Fukutomi T, Nanasawa T, Hasegawa T. Nodular mucinosis of the breast: a case report with clinical and imaging findings. Breast Cancer. 2002;9(3):261-264.

4. Sanati S, Leonard M, Khamapirad T, Eltorky MA. Nodular mucinosis of the breast: a case report with pathologic, ultrasonographic, and clinical findings and review of the literature. Arch Pathol Lab Med. 2005;129(3):e58-61.

5. Rosen PP. Rosen's Breast Pathology, ed 3. Philadelphia,
Lippincott Williams \& Wilkins, 2009.

6. Manglik N, Berlingeri-Ramos AC, Boroumand N, Eltorky M. Nodular mucinosis of the breast in a supernumerary nipple: case report and review of the literature. $\mathrm{J}$ Cutan Pathol. 2010;37(11):1178-1181.

7. Flynn V, Chisholm C, Butler D. A rare breast lesion in three patients: Furthering our knowledge on nodular mucinosis of the breast. 2011;64(2)(Supplement 1):AB38.

8. Chisholm C, Greene JF, Jr. Nodular mucinosis of the breast: expanding our understanding with an unusual case. Am J Dermatopathol. 2010;32(2):187-189.

9. Fernandez-Figueras MT, Kazakov DV, Lopez Martos R, Ojanguren I, Vila J, Ariza A. Nodular Mucinosis of the Breast in a Male: Reassessment of Diagnostic Criteria and Proposal for Its Classification as a Soft Tissue Tumor in the Myofibroblastoma and Spindle Cell Lipoma Spectrum. Dermatopathology. 2014;1:47-54.

10. Hamele-Bena D, Cranor ML, Rosen PP. Mammary mucocele-like lesions. Benign and malignant. Am J Surg Pathol. 1996;20(9):1081-1085.

11. Kempf W, von Stumberg B, Denisjuk N, Bode B, Rongioletti F. Trauma-induced cutaneous focal mucinosis of the mammary areola: an unusual presentation. Dermatopathology. 2014;1:24-28. 